\title{
Corporate Social Responsibility Reporting: A Comprehensive Picture?
}

\author{
Patricia Everaert*, Lies Bouten*, Luc Van Liedekerke ${ }^{+}$, Lieven De Moor ${ }^{++}$, Johan \\ Christiaens* \\ *Ghent University, Faculty of Economics and Business Administration, Kuiperskaai 55/E, 9000 \\ Gent, Belgium; Phone: 32926435 00, Fax: 3292643588 \\ E-mail: Patricia.Everaert@UGent.be \\ E-mail:Lies.Bouten@UGent.be \\ E-mail: Johan.Christiaens@UGent.be \\ ${ }^{+}$Center for Economics and Ethics, KULeuven, Belgium \\ E-mail: Luc.Vanliedekerke@econ.kuleuven.be \\ ${ }^{++}$Hogeschool-Universiteit Brussel \\ E-mail: Lieven.Demoor@hubrussel.be
}

\author{
Lieven De Moor \\ Onderzoeksgroep Finance, Accountancy \& Tax \\ HUBrussel \\ Stormstraat 2 \\ 1000 Brussels \\ Belgium \\ lieven.demoor@hubrussel.be
}

Please address all correspondence concerning this manuscript to: 